\title{
Sistemas de control de gestión de hospitales públicos: reflexiones para una cultura de autorregulación distribuida
}

\author{
ROBERTO CAPSTICK ${ }^{(1)}$ y MARIO IVÁN TARRIDE(2)
}

\section{INTRODUCCIÓN}

El surgimiento en Chile de los hospitales públicos autogestionados introduce un factor de incremento en su autonomía que motivan aquí algunas reflexiones, las que se espera puedan servir de ayuda al diseño y construcción de sistemas de control de gestión. Esto se realiza tomando como base algunos conceptos esenciales de la cibernética; lo que por cierto no es casual ni arbitrario, si se tiene en cuenta que ella es precisamente la ciencia de la comunicación, control y regulación de sistemas ${ }^{8}$.

Un Sistema de Control de Gestión Hospitalario (SICOGEH) se entiende aquí de manera amplia, desplazando la idea de particularidad informática-computacional con que habitualmente se les trata. Así, el planteamiento que se realiza se asocia a las funciones de regulación y control en la organización, definiéndolas y estableciendo la forma en que se deben ejercer, con relativa independencia de los dispositivos físicos de tratamiento de datos o reportes de actividad. De hecho, las funciones de regulación y control son piedra angular de toda organización, en el sentido que en ellas, entre otras funciones organizacionales, descansa buena parte de lo que le permite mantener su dinámica interna, no obstante los cambios del medio ambiente, conservando en consecuencia su identidad.

Los hospitales públicos chilenos, de particular interés aquí, son en primer lugar organizaciones de actividad humana y en consecuencia aplican en ellos todas aquellas consideraciones que han probado ser válidas en dicho contexto. Sin embargo, la población que atienden, el tipo de servicios que otorgan, los procesos que en su interior se realizan, la variedad de personas y profesiones que en ellos participan, así como el hecho de ser propiedad del Estado, hacen de los mismos un particular tipo de organización, que reclama atención especial al momento de diseñar e implementar sistemas de gestión.

Hoy, la introducción de la autogestión en los hospitales públicos constituye una oportunidad en sí misma para reflexionar - y actuar- respecto de la viabilidad de los mismos. Los niveles de autonomía, responsabilidad y discreción hospitalaria requieren hacerse evidentes en la búsqueda de más y mejores mecanismos de regulación, que permitan mejorar los desempeños de estas instituciones.

Con respecto a lo señalado, se debe considerar que la autogestión introduce varios elementos nuevos, dentro de los cuales se encuentran:

- Presupuesto directamente asignado por el Ministerio de Salud en función de lo que el hospital solicite para cumplir con la oferta de prestaciones a que lo obliga el Servicio de Salud al que pertenece.

- Facultad para diseñar su estructura organizacional de acuerdo a sus propios criterios y necesidades.

- Facultad para administrar excedentes de caja efectuando colocaciones en el mercado financiero.

\footnotetext{
(1) Ministerio de Salud. Chile. General Bulnes 51 Depto 103. Santiago. Chile.rcapstick@minsal.cl
}

(2) Universidad de Santiago de Chile. Santiago. Chile. 
- Obligación de cumplir requerimientos de calidad en instalaciones y prestaciones (acreditación).

- Facultad para comprar servicios en el mercado privado por un monto total de hasta un $20 \%$ de su presupuesto anual total.

Estas facultades, en cierta medida, entregan un mayor poder regulador a las autoridades encargadas de la administración del hospital y, al mismo, tiempo las obliga a cumplir con determinadas metas, entre las cuales una de las más relevantes es el equilibrio presupuestario.

Ciertamente, el control de gestión ha sido y es una herramienta no prescindible, sin embargo, las actuales circunstancias hacen que adquiera mayor relevancia, dado que se ha sumado a la necesidad, el incentivo de perfeccionar los métodos de control de gestión.

Por otra parte, en un ambiente de reforma en la salud, los argumentos anteriores adquieren una relevancia sustancial. De hecho, en términos generales, nuestro país ha experimentado un aumento considerable de la expectativa de vida, fundamentalmente en virtud de las políticas públicas en salud de los últimos decenios. Esto significa que conjuntamente con el aumento del promedio de edad de la población, existe un incremento en la cantidad y complejidad de las patologías que el sistema de salud debe estar en condiciones de resolver y que por su naturaleza son cada vez más costosas. Se suma, a este escenario las Garantías Explícitas en Salud, las cuales obligan al sistema a responder de acuerdo a estándares establecidos. Así, los hospitales autogestionados requerirán de nuevos sistemas de administración $\mathrm{y}$, en particular, de mejores sistemas de control de gestión, a objeto de imprimir mayor eficiencia a su actividad, permitiéndoles actuar conforme a la cantidad y calidad de prestaciones que sus beneficiarios les exijan.

\section{ORGANIZACIONES VIABLES}

Como punto de partida, es posible pensar una organización como un colectivo o agrupación humana que ha desarrollado para sí ciertas reglas de conectividad, comunicación y de toma de decisiones entre sus miembros, al punto de haberse convertido en un todo con bordes o fronteras suficientemente claras que permiten diferenciarlo de su entorno. Esa capacidad de proyectarse 'separado' del medio ambiente, indica su autonomía decisional o clausura organizacional, y en tanto le permite conservarse a través del tiempo, su viabilidad ${ }^{2}$. Es en este espacio, denominado organización, donde tiene sentido hablar de sistemas de control de gestión.

La descripción sistémica de la organización debe hacerse desde la aproximación estructural y funcional. Esto es, la organización puede ser concebida como un conjunto de subsistemas interrelacionados generando la sinergia que hace que la organización sea algo distinto a la simple suma de sus partes. A su vez, cada subsistema de la organización puede ser entendido como una totalidad compuesta por un conjunto de partes en interacción con resultado sinérgico y así sucesivamente, manifestando el principio de la recursividad estructural sistémica. Desde el punto de vista funcional, la organización puede ser concebida como una unidad simple o totalidad que realiza algo en un determinado ambiente; exhibe comportamientos, cambios de estado a través del tiempo, asociables a la organización como un todo y, recursivamente, a cada uno de sus componentes.

Desde esta perspectiva, los hospitales pueden concebirse como organizaciones que otorgan atención médica a las personas que la requieran, manteniéndolas uno o más días internadas en sus dependencias. La forma en que actualmente se estructuran los hospitales, permite distinguir subsistemas que realizan una tarea específica por la cual son reconocidos y diferenciados; se pueden identificar, por ejemplo, los diversos servicios clínicos, tales como: pediatría, ginecología, urología, entre varios otros.

Un sistema es considerado viable cuando es capaz de adaptarse y mantenerse a través del tiempo, exhibiendo una existencia relativamente separada de su medio ambiente. Esto no significa que no se encuentre en contacto con el medio o 
que el medio no contenga al sistema. Por el contrario, cuando el medio cambia el sistema puede realizar ajustes en su interior que le permiten mantener sus variables esenciales dentro de los rangos que determinan que el sistema se conserve como tal. En general, todos los organismos vivos comparten esta característica, son, por lo menos durante un cierto lapso, sistemas viables. Desde el punto de vista estructural recursivo, un sistema viable está a su vez compuesto de sistemas viables de autonomía relativa. Dicho de otra forma, los mecanismos que garantizan viabilidad a los sistemas como totalidades, se repiten en los subsistemas que los constituyen ${ }^{2}$.

En el ejemplo de los servicios clínicos, éstos pueden ser considerados sistemas viables por cuanto se replican en ellos las actividades esenciales que el hospital como un todo realiza y que le otorgan su identidad. Obsérvese que son las actividades médicas hospitalarias las que hacen esto y no aquellas que pueden ser consideradas de apoyo, como las tareas administrativas, por ejemplo la contabilidad. La esencia del hospital se juega en sus actividades primarias y no en las administrativas o meta-sistémicas que están presentes en todas las organizaciones. Perfectamente sería posible separar de un hospital un servicio clínico, como el gineco-obstétrico, por ejemplo, y constituir a partir de él una nueva organización hospitalaria. Tendría una única especialidad, como el Instituto de Neurocirugía de Santiago, pero ciertamente sería un hospital. Esto mismo no ocurre con el Departamento de Contabilidad, dependiente de la Subdirección Administrativa, ya que si se le separa, no llegará a convertirse en hospital.

En general, todos los sistemas viables comparten una característica particular, a saber: disponen de un 'dispositivo' que les permite efectuar cambios internos para hacer frente a las perturbaciones provenientes de su medio ambiente y que podrían sacar a las variables esenciales de los rangos que demarcan el que el sistema no sea destruido. Por ejemplo, frente a una variación violenta de la temperatura en el medio ambiente, ciertos organismos vivos, por medio de redes neuronales o químicas, pueden hacer que sus vasos sanguíneos periféricos se expandan o contraigan y con ello pueden regular su temperatura interior, manteniéndola constantemente alrededor de los $36 \mathrm{C}^{\circ}$, en el caso de los seres humanos. De hecho, a este dispositivo se le denomina 'regulador'.

En particular, la viabilidad de los hospitales públicos chilenos a través de los años puede explicarse desde la propiedad sistémica de la clausura organizacional antes señalada. En este caso, el cierre de los procesos decisorios para el mantenimiento de los mismos, no se verifica a nivel de hospital y tampoco de servicio de salud, entidad de la cual el hospital depende. Es el Estado quien posee esta propiedad de clausura, distribuida entre el poder Ejecutivo y el Legislativo. De este modo, el prestador público está obligado a brindar tantas prestaciones como sus beneficiarios le demanden hasta agotar su presupuesto e incluso ir más allá, por medio del endeudamiento con sus proveedores. Finalmente, es el Ministerio de Hacienda el que se encarga de 'reponer' los recursos para la continuidad de la operación del hospital. Así, la viabilidad de los hospitales públicos no ha estado comprometida, de manera directa, con el nivel de autonomía de decisiones que ellos tienen.

\section{REGULACIÓN DE VARIABLES ESENCIALES}

Cada una de las características que observamos de un fenómeno y que conforman un sistema corresponde a lo que formalmente se denomina variable. Un sistema, entonces, está compuesto por un conjunto de variables, las cuales experimentan cambios dentro de ciertos rangos a lo largo del tiempo.

Una variable esencial ${ }^{1}$ es aquella que en caso de traspasar los umbrales de normalidad de su comportamiento, determina la destrucción del sistema. Así, entre las observaciones que se hacen de un fenómeno y que son formalmente variables, habrá algunas que serán esenciales y otras no.

Para el ejemplo anterior relativo a la temperatura corporal, es posible observar que ante 
un descenso en la temperatura y el consiguiente enfriamiento de la sangre, el cuerpo responde contrayendo los vasos sanguíneos. No actúa directamente sobre la sangre sino que lo hace a través de otra variable como son los vasos periféricos, dejando con ello constancia de la existencia de un canal comunicacional entre ambas variables. Además, la dilatación relativa de los vasos sanguíneos puede ser considerada como una variable de regulación que el organismo puede modificar a voluntad y con ello lograr que otra variable también cambie. No puede actuar directamente sobre la temperatura del medio o de la sangre, pero puede lograr su objetivo variando la dilatación mencionada, dicho de otra forma, puede variar el estado del sistema y conservar su existencia como tal, al movilizar la variable de regulación; dilatación de los vasos sanguíneos. Por lo tanto, los sistemas pueden definirse por un conjunto de variables, entre las cuales se encuentran las esenciales para la viabilidad del sistema, otras consideradas útiles para la regulación del mismo, y aquellas que, aun cuando forman parte constitutiva del sistema, tienen menor relevancia para su viabilidad.

La regulación de las variables esenciales puede explicarse desde la "ley de variedad obligada" de Ashby', según la cual para hacer frente a los cambios que tienen lugar en el medio ambiente, un sistema debe tener la capacidad de realizar por lo menos la misma cantidad de variaciones que las que evidencia el medio ambiente y no menos. Expresado de otra forma, el sistema está obligado a disponer de tanta capacidad de variación para conservar su viabilidad como variedad exhiba el medio ambiente. Por cierto, esta ley marca una frontera teórica o ideal, que la factibilidad real reduce a niveles que se ubican muy por debajo de lo necesario.

Si un hospital, como ocurre en los meses de invierno en Santiago, enfrenta un aumento de demanda y sus niveles de producción no alcanzan los requeridos para satisfacerla, la administración del mismo, entendida como dispositivo de regulación, puede contratar horas extraordina- rias y/o nuevo personal, aumentar el rendimiento de sus equipos si es posible, incorporar nuevos recursos físicos y de infraestructura, comprar temporalmente servicios a terceros, entre otras medidas.

Una condición mínima de viabilidad indica que el sistema debe disponer de dispositivos de regulación que le permitan detectar cambios dentro del sistema y actuar en consecuencia para la conservación del mismo; estos cambios pueden provenir tanto de las variables esenciales como del resto de las variables. Este comportamiento reactivo puede ampliarse a uno anticipatorio, si el sistema posee dispositivos que le permiten detectar cambios en el medio ambiente que podrían llegar a afectarlo, ante los cuales actuaría preventivamente, evitando así la afectación de sus variables internas y manteniendo la viabilidad del sistema. Más aún, un tercer tipo de regulación sería posible, homóloga al concepto de promoción de la salud en las personas esto es, un sistema con mecanismos que le permitan desarrollar e incrementar aquellos comportamientos que favorecen su adaptación, viabilidad y mejor desempeño. Es decir, lograr el control de las variables que determinan su comportamiento.

Las variables esenciales que se definen para un sistema viable pueden modificar su comportamiento dependiendo del tipo de regulación que disponga el sistema: por error, intermediada y/o anticipada ${ }^{1}$. Entonces, el dispositivo de regulación del sistema debe distinguir claramente entre estas tres situaciones con el objeto de actuar según corresponda.

En el caso de la regulación por anticipación, el dispositivo regulador es capaz de percatarse de la aproximación de un cambio o perturbación en el medio ambiente y de prever el desequilibrio que ese cambio ocasionaría al interior de la organización. De este modo, los sensores informan a los efectores para que ejecuten aquellas acciones correctivas, previamente definidas o contingentemente ideadas, que de manera anticipada evitarán el desequilibrio en la organización. En la regulación por intermediación, los sensores se 
informan de cambios o perturbaciones provenientes del medio ambiente, sólo después que éstas han afectado a alguna variable organizacional, pero que no tiene la categoría de esencial. El regulador actúa en consecuencia, enviando a través de los canales efectores las medidas correctivas que protegerán a las variables esenciales. Por último, en la regulación por error, las perturbaciones medioambientales alcanzan a las variables esenciales, sacándolas de su comportamiento normal. Esta alteración es recogida por los sensores del sistema regulador, comunicada a su nivel decisional que determina las acciones a realizar, para luego ser ejecutadas a través de los dispositivos efectores. En este caso se actúa con atraso respecto de la perturbación, lo que significa que se ha producido una cierta cantidad de daño o pérdida en el sistema.

De estos tres tipos de regulación, la anticipada permitiría alcanzar mejores resultados, no sólo porque se adelanta a las perturbaciones, sino porque además deja espacio para actuar, en caso de falla, con la regulación por intermediación y por error. En consecuencia, un SICOGEH debería orientarse, tanto como se pueda, hacia la regulación por anticipación, pero sin descuidar la redundancia protectora que provee disponer de los otros dos tipos de regulación. Para el ejemplo anterior de las enfermedades respiratorias, la regulación anticipada que realiza un hospital de Santiago puede verse en las estrategias desplegadas para hacer frente a la mayor demanda por atención que se tiene durante los meses de invierno (campaña de invierno): es necesario disponer de buenos sistemas de predicción de demanda, según los cuales comprometer por adelantado un aumento de las horas profesionales y de apoyo contratadas, así como de los equipos y materiales requeridos para dar satisfacción al aumento de demanda esperada. En este caso particular, no parece conveniente usar la regulación por error, dado que significaría informarse una vez que la variable esencial 'número de camas respiratorias disponibles' se haya salido de su banda de normalidad, y sólo entonces actuar; en ese momento ya se tendrían pacientes hospitalizados en camillas, en los pasillos del hospital.

Desde esta perspectiva, toda organización viable dispone de una estructura cuya conectividad permite la comunicación de información y la implementación de medidas reguladoras de acuerdo a las decisiones diseñadas y tomadas por sus equipos directivos.

Consecuentemente entonces, un SICOGEH debe concebirse formando parte inherente de la conectividad de un hospital, pieza fundamental en la permanente tarea de modificar la estructura del mismo, con el objeto de hacerlo viable a pesar de estar inmerso en un medio ambiente esencialmente cambiante.

La viabilidad organizacional depende de la calidad de sus mecanismos reguladores. A manera de ejemplo, se puede considerar una organización cualesquiera en la que mientras su actividad general cae, la unidad de abastecimiento sigue realizando adquisiciones, aumentando el stock y también las deudas con sus proveedores. Si este proceso continúa, sin un sistema que tenga simultáneamente en observación la disponibilidad de efectivo, los niveles de stock de materias primas y el nivel de ingresos operacionales, 1legará el momento en que se deberá cumplir con las obligaciones financieras con los proveedores, pero no habrá liquidez disponible para hacerlo, dado que han caído los ingresos operacionales. Los acreedores pueden solicitar la quiebra, la organización es entonces intervenida, se liquidan sus activos, se cancelan las obligaciones y la organización deja de existir como tal. En suma, la caída en las operaciones, en ausencia del dispositivo regulador que ajusta las variables esenciales, determina la destrucción del sistema viable. Del dispositivo de regulación se espera provengan las decisiones que varíen los niveles de inventarios y la deuda con los proveedores, deteniendo las adquisiciones cuando la variable ingresos de operación cae y de esta forma se mantengan las variables dentro de rangos aceptables, es decir, se logre la viabilidad de la organización.

Con relación al ejemplo anterior, una diferen- 
cia importante puede establecerse entre las organizaciones privadas y los hospitales públicos; estos últimos disponen de una segunda instancia garante de viabilidad ante fallas en sus propios mecanismos reguladores: el Estado. Es éste el que produce la clausura decisional cuando se vulnera una necesidad de la población consagrada en la Constitución y que, en consecuencia, está obligado a proveer.

Pero no sólo la regulación asegura viabilidad, es necesario conocer los objetivos perseguidos por la organización. Cibernéticamente, la función control es aquella que se encarga de fijar los objetivos al sistema ${ }^{1}$; esto es, fija los valores de normalidad de las variables esenciales. Se destaca su concepción completamente distinta a la de algunas escuelas de pensamiento administrativo que consideran a control como una función de vigilancia y sanción/premiación de carácter coercitivo. En tanto que la función reguladora es aquella que se encarga de informarse de los cambios internos y externos al sistema y actuar de acuerdo a estrategias previamente definidas o diseñadas, para el logro de los objetivos perseguidos o el mantenimiento de las variables esenciales dentro de sus rangos de comportamiento normal. Es el sistema regulador el que realiza las mediciones de las variables esenciales y el que las compara con los objetivos impuestos por control. Esta tarea, como se señaló anteriormente, puede ser de tipo anticipatoria, intermediada y por error.

\section{RECURSIVIDAD DE SISTEMAS VIABLES}

Como se señaló anteriormente, de acuerdo al principio de recursividad, la organización en tanto sistema viable está compuesta por subsistemas que a su vez también son sistemas viables y así sucesivamente. Luego, todos y cada uno de estos sistemas deberán realizar funciones de regulación y control, para lo cual requieren, a su vez, de sistemas de control de gestión en cada uno de ellos. Esto significa, por ejemplo, que en un hos- pital el SICOGEH necesariamente debe ser concebido de manera integrada/distribuida, en que cada Servicio Clínico dispone de un subsistema de control de gestión, que a su vez forma parte del sistema de control de gestión del hospital. En otras palabras, el SICOGEH es de todos y cada uno de los niveles y unidades del hospital y no sólo de los niveles directivos superiores.

En la práctica, el control cibernético de un hospital se establece recursivamente desde la dirección hacia los diferentes servicios clínicos que lo conforman, al fijar los objetivos y sus rangos de normalidad. Esta tarea se realiza a través de ejercicios estratégicos y de planificaciones y programaciones anuales. También ocurre a través de los estándares que provienen de los niveles jerárquicos superiores, sean estos Servicios de salud y/o Ministerio de Salud. La regulación, en tanto, será realizada por los encargados de la gestión hospitalaria desde el nivel directivo superior hasta el menor nivel operacional al interior de los servicios clínicos.

Para que la tarea reguladora se realice de manera efectiva en el logro de la viabilidad, el SICOGEH debe satisfacer como mínimo tres condiciones necesarias: variables esenciales identificadas para cada nivel recursivo e interrelacionadas, rangos de comportamiento normal u objetivos esperados para cada variable y periodos o momentos oportunos de medición para cada una de ellas. Sin estos tres elementos, no es posible que el sistema cumpla con su razón de ser y brinde la utilidad que de él se espera. El sistema debe operar cabalmente en los términos de su diseño, el cual será satisfactorio sólo si es realizado en función del concepto de viabilidad. La complejidad técnica del mismo, el qué tan sofisticado sea o su exactitud, no son parámetros en modo alguno adecuados para medir su calidad como tal.

Al estar cada sistema viable inmerso en un medio ambiente que lo contiene, cualquier subsistema de una organización estará también contenido en un medio ambiente, que en parte estará conformado por factores externos a la organiza- 
ción y en parte por componentes de la organización misma, y deberá regular su actuación para mantenerse viable ante las variaciones que experimenten tanto los primeros como los segundos. Así, por ejemplo, la unidad de imagenología de un hospital deberá responder a los requerimientos que le formulen las otras unidades hospitalarias, pero no sólo eso, sino que también deberá responder a las variaciones del medio externo como pueden ser los cambios en los mercados de insumos que hagan incrementar sus costos, o cambios tecnológicos que al incorporarlos puedan aumentar su capacidad de producción.

De esta forma, un SICOGEH es mucho más que un sistema de registro de ciertos aspectos de la actividad de cada unidad organizacional. De hecho, es una parte activa de la administración de cada una de ellas que le permite determinar los cambios que cada subsistema debe realizar en su interior para conservar sus variables esenciales dentro de los rangos establecidos y lograr los objetivos perseguidos.

Modelar un hospital como sistema viable recursivo permite enfrentar de mejor forma la variedad de cambios que se producen en el medio ambiente. La idea central es que cada subsistema viable hospitalario disponga de la autonomía necesaria, evitando la intervención de los sistemas superiores que los contienen y, de esta forma, liberar al sistema viable superior del esfuerzo que significaría regular al sistema completo en forma centralizada. De hecho, resultaría casi imposible que una regulación centralizada lograra la necesaria variedad de respuestas que se requiere o, cuando menos, que lo lograra con el mismo grado de eficiencia que un sistema recursivo.

Así, el quiebre del hospital en servicios clínicos, que es donde se materializan los procesos de transformación que le dan identidad a un hospital, permite distinguir a lo menos dos niveles recursivos. Y, dado que cada servicio clínico es un sistema viable, entonces cada uno ejecutará las funciones de regulación y control que le correspondan para el mantenimiento y desarrollo del mismo.
Si cada subsistema viable es capaz de dar respuestas satisfactorias a las perturbaciones de su medio ambiente, entonces ¿cuál es la tarea del sistema viable que los contiene? Dos aspectos deben tenerse en cuenta en este caso: las 'sinergias' y las 'emergencias' ${ }^{3}$. Las primeras, dicen relación con aquellas propiedades que surgen como consecuencia de las interacciones no lineales de los componentes de un sistema; 'el todo es algo más que la simple suma de sus partes'. El nivel recursivo superior es responsable de que estas sinergias ocurran, esto es, que el resultado perseguido efectivamente se obtenga al poner en relación sus distintos componentes de una cierta manera. Así, la forma en que los diferentes subsistemas viables interactúan —se coordinan - para el logro del objetivo, es de su responsabilidad. Pero no sólo debe preocuparse de que se logre el resultado esperado, también debe atender a aquellas situaciones especiales, inesperadas, imprevistas, denominadas 'emergencias', que surgen por el conocimiento parcial y contingente que se tiene de cualquier sistema, y para las cuales no tiene estrategias de enfrentamiento predefinidas, obligándolo a disponer de recursos de imaginación e inteligencia que diseñen estrategias específicas para enfrentarlas en el momento que se producen.

En consecuencia, una tarea fundamental del sistema superior es la coordinación de los subsistemas viables que lo constituyen, con el objeto de lograr cohesión interna. Conjuntamente, debe hacerse cargo de regular las perturbaciones que lo afectan a su nivel jerárquico, ya sea que provengan de su medio ambiente externo, interno, o de sí mismo.

\section{ORGANIZACIÓN Y MEDIO AMBIENTE}

Un "trozo de realidad" puede ser observado de distintas formas por un mismo observador o de distintas formas por cada uno de los miembros de un grupo de observadores. Se puede convenir entonces que existen variadas formas 
de percibir la realidad y que para cada una de esas formas se tiene un criterio de distinción de una entidad respecto del medio en el cual se encuentra. De acuerdo con lo señalado anteriormente, un criterio general y útil para distinguir una organización, es considerarla un sistema viable que mantiene una existencia separada de su medio ambiente. La propia organización diseña e implementa estrategias para hacer frente a las perturbaciones provenientes del medio, logrando así su viabilidad.

De lo anterior es posible derivar que la organización es todo aquello que conforma un sistema propio, exclusivo, de toma de decisiones y de ejecución de las mismas, y que no le pertenece a ningún otro ente: la organización, en su calidad de tal, es un sistema cerrado de toma de decisiones o dicho de otra forma, dispone de clausura decisional y operacional ${ }^{6}$. Consecuentemente, todo lo demás, conforma el medio ambiente. Más difícil es definir este último en términos específicos. Una forma sintética y adecuada para describirlo viene dada por el concepto de transformación ${ }^{1}$. En efecto, la organización es un sistema que toma elementos del medio y los transforma haciendo que esos elementos cambien de estado y/o forma: la organización entonces puede ser vista como una caja negra que transforma entradas (insumos) en salidas (productos). El quehacer e identidad de clase de la organización queda definido por el conjunto de transformaciones que ella realiza. En tanto que todos aquellos factores externos a la organización y que dicen relación con su quehacer, permiten distinguir su entorno relevante, motivo de atención permanente para el cuidado de la viabilidad de la organización. En el caso de un subsistema viable, la definición de medio ambiente relevante, al igual que en el caso de la organización en su conjunto, está determinado por las transformaciones que esa unidad en especial realiza. Así, poner la atención sobre el proceso de transformación, permite reducir la descripción del medio ambiente a un nivel manejable. De lo contrario, la definición es tan amplia que pierde por completo su utilidad.
Cada subsistema de la organización contribuye al logro de los objetivos generales estratégicos de ella. Esto lo hacen al participar activamente en el diseño de formas factibles de lograr los objetivos que les competen. El conocimiento que el sistema como totalidad tiene de su medio ambiente relevante, así como el de cada subsistema, permite diseñar estrategias reales para el logro de los objetivos perseguidos; dicho de otra forma, todo sistema y subsistema diseña conforme el medio ambiente restringido que percibe, hecho que permite acotar y ajustar estrategias y decisiones, pero que también implica dotar de inteligencia y flexibilidad a la organización para hacer frente a aquellas perturbaciones que el diseño estratégico no consideró y que ciertamente se producirán poniendo en jaque la viabilidad de la organización y de cada uno de los subsistemas que la componen.

Por otra parte, debe tenerse en consideración que los objetivos estratégicos son metas de mediano a largo plazo y que la administración de un sistema viable, en cualquier nivel recursivo, debe cumplir también con objetivos de corto plazo o inmediatos; por cierto, ambos están íntimamente ligados. Por lo tanto el SICOGEH debe incluir, dependiendo del nivel recursivo en que se encuentre, indicadores asociados a largo, mediano y corto plazo, permitiendo derivar unos de otros. Este principio general permite economía de recursos informáticos y facilita la consistencia de la información. No obstante, es posible que los datos estratégicos y operativos deban procesarse en forma separada, debido a las condiciones particulares que puede adoptar la información en cada caso. Así, lo señalado debe considerarse una forma deseable pero no indispensable en el procesamiento de información.

\section{EL SISTEMA DE CONTROL DE GESTIÓN HOSPITALARIO}

El SICOGEH, como hasta aquí se ha descrito, que concibe a la organización como un sistema 
viable conformado a su vez, de acuerdo al principio de recursividad, por sistemas viables dentro de sistemas viables, presupone necesariamente que su función sea una actividad esencial y propia de cada uno de los sistemas viables al interior del hospital. De hecho, el SICOGEH, más allá de ser una forma de reporte de los niveles de actividad de una unidad organizacional, según el cual se establece su grado de cumplimiento de ciertos parámetros de actuación, es una función que entrega a la administración de la unidad una visión global y completa de la viabilidad efectiva de la misma. De esta forma, al estar el SICOGEH distribuido a través de toda la organización, facilita la integración de todos y cada uno de sus niveles recursivos.

Como se ha señalado, viabilidad de un sistema significa que sus variables esenciales se encuentran, en un período de tiempo definido, dentro de un rango de valores específicos considerados normales y si alguna de estas variables sale de ese rango durante un tiempo suficiente, entonces el sistema deja de existir como tal.

Para un hospital público ¿cómo se definen sus objetivos, variables esenciales y rangos o umbrales de normalidad? De hecho, como se señaló anteriormente, a diferencia de una entidad privada, un sistema viable de carácter estatal no deja de existir como tal por el hecho de que sus variables económicas se encuentren fuera de rango. La autonomía de un hospital público se clausura a nivel de Estado, dado que este último está obligado a responder ante las necesidades y derechos de la población. Obsérvese al respecto las importantes deudas históricas que mantienen muchos hospitales del país con sus proveedores. Por cierto, el Estado puede dejar de proveer un bien o servicio, cuando por ejemplo el usuario ya no requiere de él o porque el sector privado ha comenzado a servirlo y se ha observado que adquirirlo en el mercado resulta más conveniente que producirlo. Así, el mayor objetivo estratégico de un hospital público se iguala al del Estado; esto es, atender las necesidades de salud de las personas.

El contexto social, cultural, político, económico, tecnológico y epidemiológico del país res- tringe el diseño de identidad de los hospitales e indica la forma de proceder para el desarrollo de sus tareas. Hoy, las definiciones se realizan de manera vertical, de arriba hacia abajo, y emanan de las políticas, estrategias, objetivos y metas de salud diseñados en el nivel central. Estos insumos determinan recursivamente las obligaciones de los niveles intermedios constituidos por los servicios de salud y del nivel básico u operativo donde se encuentran los hospitales y la atención primaria.

Nuevamente parece bueno recordar que dado un sistema viable que contiene subsistemas viables, estos últimos subordinan su viabilidad a la viabilidad del sistema mayor que los contiene. Esto significa que la determinación de las variables esenciales de los subsistemas depende de las variables esenciales del sistema mayor que los contiene. Pero no sólo eso, esta recursividad de sistemas viables hace surgir la función de coordinación, dado que ya no es suficiente con que cada uno de los subsistemas cumpla con sus objetivos, expresados en términos de mantener sus propias variables esenciales dentro de los rangos de normalidad determinados, sino que además deben funcionar coordinadamente, tarea propia del sistema que los gobierna.

Cada hospital, en el marco que le impone el servicio de salud y la red asistencial a la cual pertenece, define sus objetivos estratégicos, haciendo uso de algún método apropiado para ello. Luego, cada uno de los subsistemas viables que componen el hospital —servicios clínicos-, definen a su vez sus propios objetivos en función de los establecidos para el hospital como un todo. Conjuntamente y asociado a los objetivos específicos de cada subsistema viable, se definen las variables esenciales con sus bandas o umbrales de normalidad que garanticen el cumplimiento de los objetivos perseguidos. Además, los componentes de cada subsistema viable deben establecer acuerdos de coordinación para su operación conjunta, tarea que también debe ser realizada por todos los subsistemas viables que componen el hospital. Durante este proceso el hospital no sólo se infor- 
ma de los objetivos estratégicos provenientes de los niveles superiores, sino que también participa activamente en la formulación de sus propios objetivos y estrategias. La falta de participación parece ser la principal amenaza para el logro de los mismos, permitiendo la aparición de apatía, desinterés, desidia y otras actitudes negativas entre las personas.

Los valores que toman las variables esenciales pueden ser observados a distintos intervalos, dependiendo del comportamiento de ellas. Por cierto, no es razonable observar una variable cada treinta días, si la velocidad de cambio en su comportamiento es a tasa diaria. Poco interés también tendría medir una variable día a día si su tasa de cambio es anual. En consecuencia, las frecuencias de medición deben quedar claramente establecidas en función de la velocidad de cambio estimada. Siguiendo con un ejemplo anterior, el número de camas disponibles en un hospital parece ser una variable a monitorear día a día, y no a intervalos mensuales o anuales.

Se debe definir para el sistema mayor un conjunto de variables esenciales que se refieran a la medición de la coordinación de la actividad de los subsistemas que lo conforman. Esto hace necesario definir variables de resultado finales para el sistema mayor y variables de coordinación que expliquen las variaciones en las primeras. Un caso actual típico puede ser el que se presenta entre un servicio clínico y una unidad de apoyo diagnóstico: ambas pueden, en un momento dado, mostrar su variables esenciales dentro de los rangos deseados, pero es posible que la relación entre los estados de una y otra unidad no se encuentren en concordancia, especialmente en cuanto a la oportunidad de los resultados para la determinación de los diagnósticos. Así, cada nivel recursivo deberá responsabilizarse de la coordinación de los subsistemas que lo conforman, evitando la pérdida y maximizando el nivel de ejecución del conjunto.

La estructura del SICOGEH se refiere al conjunto y disposición de los componentes constituyentes del mismo. Básicamente, como ya se ha señalado, las unidades encargadas del control de gestión serían las mismas que conforman la organización; esto es, los distintos sistemas viables que la componen. Nótese que desde esta perspectiva no se requiere una unidad específica, independiente, que realice tareas de control de gestión, materializando así un real control de gestión distribuido.

De la misma forma, los responsables del SICOGEH serían los mismos encargados de la gestión de cada uno de los sistemas viables que existan. Esto debido a que, según se ha señalado, la función de control de gestión no se entiende como una función específica y separada de las demás, sino que como una parte integrante e indispensable de la administración misma.

Bajo estas condiciones, el control de gestión en cuanto función, utilizaría los mismos recursos humanos y materiales que utiliza la administración de cada sistema viable. Los recursos específicos que se requerirían, estarían orientados particularmente al procesamiento de información. De esta manera, la obtención de los datos pertinentes y el procesamiento de los mismos, son los dos elementos fundamentales que determinarían los recursos humanos, el hardware y software necesarios, que cada sistema viable debería destinar a la realización de la función.

Por cierto, tanto la obtención de los datos como el procesamiento de los mismos, tendrán características propias en cada caso en particular. Puede requerirse personal a tiempo completo o con dedicación parcial; puede ser necesario o no, hardware y/o software sofisticado. Sin embargo, el único principio inviolable es que la información acerca del comportamiento de las variables esenciales de cada sistema y los rangos de normalidad establecidos, sean confiables y se encuentren a disposición de la administración de los subsistemas viables y de la organización en general, en los tiempos de observación determinados.

Por otra parte, es necesario destacar que un SICOGEH como el aquí propiciado, requiere de una comprensión sui generis del mismo y del proceso administrativo en general. En palabras de 
Ashby "una regulación perfecta... hace posible un control completo" del sistema y para que esto ocurra es necesario que se satisfaga la ya señalada "ley de variedad obligada", esto es, que el regulador tenga tanta variedad, como variedad perturbadora exista en el medio ambiente: he aquí la frontera de la inmortalidad. Es posible observar, en un cierto horizonte temporal, organizaciones exitosas en adaptarse y conservarse, así como otras que no fueron capaces de hacerlo y desaparecieron. Si la variedad perturbadora medioambiental es siempre mucho mayor que aquella que pueda generar el mejor de los reguladores que se pueda construir; entonces, es posible afirmar que toda organización está condenada a desaparecer en algún momento del tiempo. En consecuencia, el mejor regulador a que se puede aspirar, dadas las circunstancias, es aquel que exhiba la mayor cantidad posible de variedad, para hacer frente a la variedad perturbadora del medio. La propuesta de un sistema distribuido para los hospitales, parece entonces una mejor alternativa que uno centralizado; cada componente de la organización, por muy menor que parezca, debe ser visto como un integrante más del sistema regulador y en consecuencia del SICOGEH.

No obstante lo anterior, se considera que un proceso de instalación de un SICOGEH podría hacerse paulatinamente, acompañado de un proceso de ajuste cultural en toda la organización y liderado desde una unidad especializada capaz de dirigir el proceso en su totalidad, tanto en la fase de desarrollo del modelo como durante su implantación, de acuerdo a los principios aquí establecidos. No responde al prototipo de las actuales unidades o gerencias de control de gestión, sino más bien a una entidad de cambio organizacional de vida finita, cuyo cese estaría dado por el logro del ajuste organizacional perseguido, esto es, una cultura de autorregulación distribuida.

Por último, es necesario precisar que no se descarta aquí ningún instrumento que ayude en la tarea de diseño e implementación de un SICO$\mathrm{GEH}$, de modo que herramientas como la Planificación Estratégica ${ }^{4}$ y los Modelos de Control de Gestión ${ }^{5}$, deben ser considerados y usados según lo aconsejen las circunstancias.

\section{CONCLUSIONES}

Las reflexiones cibernéticas planteadas conducen, entre otras cosas, a considerar los SICOGEH en un sentido amplio que los ubica en el corazón mismo de la gestión hospitalaria y no sólo como una accesorio más de la gestión. Deben ser concebidos como sistemas que permitan una gestión distribuida, descentralizada, con indicadores de corto, mediano y largo plazo, adecuados a las variables esenciales distinguidas en los servicios clínicos del hospital y cuyo monitoreo ocurre según la tasa de cambio de las mismas.

El control distribuido del SICOGEH mejoraría la eficacia reguladora, pero ciertamente no eliminaría la posibilidad de destrucción del sistema ante perturbaciones para las cuales no se disponga de variedad neutralizadora.

De acuerdo con lo anterior, se considera que el SICOGEH debe disponer de la mayor cantidad de variedad posible y factible de implementar, de modo que garantice la realización de regulación por anticipación, por intermediación y por error, así como también la coordinación. Cuando las estrategias de regulación se acaban, es necesario disponer de mecanismos de imaginación e inteligencia organizacional que generen otras nuevas, en especial ante situaciones emergentes.

Los hospitales y sus servicios clínicos son considerados sistemas viables, en tanto ellos pueden mantener una existencia de autonomía relativa respecto de su medio ambiente. La actividad central de un hospital ocurre en sus servicios clínicos, son estos los que le otorgan su identidad. Es consecuencia, es para ellos que deben identificarse las variables esenciales, sus bandas de comportamiento normal y los dispositivos reguladores para hacer frente a las perturbaciones del medio, tanto interno como externo.

Los SICOGEH contribuirán al logro de los objetivos perseguidos, en tanto sus mecanismos detectores de perturbaciones y efectores de es- 
trategias reguladoras, sean de buena calidad y cantidad suficiente, disponiendo de canales de comunicación expeditos que faciliten el flujo de información.

Desde el punto de vista del diseño e implementación del SICOGEH, es posible señalar las siguientes consideraciones:

- Todo el personal del hospital debería conocer los objetivos perseguidos por el mismo, en especial los correspondientes a su unidad y la vinculación entre ambos.

- Cada unidad constitutiva del establecimiento debería participar activamente en el diseño e implementación de los procesos necesarios para alcanzar los objetivos.

- Cada servicio clínico debería llevar a cabo los acuerdos con las unidades organizacionales que conforman su medio ambiente interno en términos de la forma en que se relacionarán en la operación.

- Cada servicio clínico debería definir sus variables esenciales, los rangos o umbrales de viabilidad para cada una de ellas y los períodos de medición. El criterio básico para definir estos últimos, es la probabilidad de que entre una medición y otra, la variable pueda fluctuar y eventualmente salir de su rango de normalidad, comprometiendo la viabilidad del sistema.

- Cada servicio clínico debería definir las variables que se relacionan con los objetivos estratégicos de su responsabilidad y que contribuyen a satisfacer los objetivos estratégicos del hospital como totalidad.

- Se debería diseñar un sistema informático integrado/distribuido que capte y procese los datos requeridos, que calcule los indicadores asociados a las variables esenciales y estratégicas en cada período de medición y emita los reportes que efectivamente apoyen la gestión de las distintas unidades y del hospital en general.

Finalmente, se espera que las ideas expuestas contribuyan al diseño e implementación de sistemas de control de gestión cada vez más adecuados a las necesidades de los hospitales públicos del país.

\section{REFERENCIAS}

1. ASHBY W. ROSS. 1972. Introducción a la Cibernética. Ediciones Nueva Visión. Buenos Aires.

2. BEER SATAFFORD. 1981. Brain of the Firm. 2da. Edición, Wiley, Chichester.

3. CHECKLAND, PETER. 1981. Systems Thinking, Systems Practice. Ed. John Wiley \& Sons.

4. HAX ARNOLDO C., MAJLUF NICOLÁS S. 1997. Estrategia para el Liderazgo Competitivo. Editorial Dolmen, Argentina.

5. KAPLAN ROBERT S., Norton David P. 1997. El Cuadro de Mando Integral (The Balanced Scorecard). Ediciones Gestión 2000, Barcelona.

6. LE MOIGNE, JEAN-LOUIS. 1990. La Modélisation des Systèmes Complexes. Collection Afcet Systèmes, Dunod. Paris, France.

7. PORTER MICHAEL E. 1989. Ventaja Competitiva. Editorial Continental, México.

8. WIENER, NORBERT. 1948. Cybernetics. John Wiley and Sons.

Recepción: 23 de mayo de 2008 Aprobación : 4 de septiembre de 2008

Usted puede comentar éste y otros artículos publicados en la Revista Chilena de Salud Pública, enviando un correo electrónico a revistasp@med.uchile.cl 